Physics

Physics Research Publications

\title{
Magnetoconductance oscillations in graphene antidot arrays
}
T. Shen
Y. Q. Wu
M. A. Capano

L. P. Rokhinson

L. W. Engel

P. D. Ye 


\title{
Magnetoconductance oscillations in graphene antidot arrays
}

\author{
T. Shen, ${ }^{1,2}$ Y. Q. Wu, ${ }^{1}$ M. A. Capano, ${ }^{1}$ L. P. Rokhinson, ${ }^{2}$ L. W. Engel, ${ }^{3}$ and P. D. Ye ${ }^{1, a)}$ \\ ${ }^{1}$ School of Electrical and Computer Engineering and Birck Nanotechnology Center, \\ Purdue University, West Lafayette, Indiana 47907, USA \\ ${ }^{2}$ Department of Physics, Purdue University, West Lafayette, Indiana 47907, USA \\ ${ }^{3}$ National High Magnetic Field Laboratory, Tallahassee, Florida 32310, USA
}

(Received 10 July 2008; accepted 4 September 2008; published online 22 September 2008)

Epitaxial graphene films have been formed on the $\mathrm{C}$-face of semi-insulating $4 \mathrm{H}$-SiC substrates by a high temperature sublimation process. Nanoscale square antidot arrays have been fabricated on these graphene films. At low temperatures, magnetoconductance in these films exhibits pronounced Aharonov-Bohm oscillations with the period corresponding to magnetic flux quanta added to the area of a single antidot. At low fields, weak localization is observed and its visibility is enhanced by intervalley scattering on antidot edges. At high fields, we observe two distinctive minima in magnetoconductance, which can be attributed to commensurability oscillations between classical cyclotron orbits and antidot array. All mesoscopic features, surviving up to $70 \mathrm{~K}$, reveal the unique electronic properties of graphene. (C) 2008 American Institute of Physics. [DOI: 10.1063/1.2988725]

Antidot arrays are interesting structures to study because of their transport properties in conducting electronic materials. ${ }^{1}$ Antidots can be regarded as groups of the imposed scatters that limit the ballistic transport or the mean free path of carriers. Antidot arrays can also be considered as an ensemble of Aharonov-Bohm (AB) rings connected together. ${ }^{2,3}$ Moreover, antidot arrays are ideal for investigating quantum coherent effects and phase coherence length of carriers. Graphene, a monolayer of carbon atoms tightly packed into a two-dimensional (2D) hexagonal lattice, has recently been shown to be thermodynamically stable and exhibits astonishing transport properties, such as an electron mobility of $\sim 15000 \mathrm{~cm}^{2} / \mathrm{V} \mathrm{s}$ and electron velocity of $\sim 10^{8} \mathrm{~cm} / \mathrm{s}$ at room temperature. ${ }^{4-9}$ However, graphene is semimetallic and thus not suitable for most electronic and optoelectronic applications, which require a semiconductor with a specific finite bandgap. Antidot arrays impose lateral potential barriers that could create a bandgap in graphene, ${ }^{10}$ similar to the creation of the energy gap by introducing lateral periodic potentials from positive ion cores in a real semiconductor crystal. In this letter, the magnetotransport properties of this kind of nanostructured antidot arrays in an epitaxially grown graphene film on $\mathrm{SiC}$ are investigated. Pronounced $\mathrm{AB}$ oscillations, weak localization, and commensurability oscillations are observed directly related to electrical properties of epitaxial graphene films such as mean free path and phase coherence length.

The advantage of epitaxially grown graphene for nanoelectronic applications resides in its planar $2 \mathrm{D}$ structure that enables conventional top-down lithography and processing techniques. ${ }^{7-9}$ The graphene films in this letter are grown on the carbon face of semi-insulating $4 \mathrm{H}$-SiC substrates in an Epigress VP508 SiC hot-wall chemical vapor deposition reactor. The off-cut angle of the substrate is nominally $0^{\circ}$. Prior to growth, substrates are subjected to a hydrogen etch at $1600{ }^{\circ} \mathrm{C}$ for $5 \mathrm{~min}$, followed by cooling the samples to below $700{ }^{\circ} \mathrm{C}$. After evacuating hydrogen from the system, the

\footnotetext{
a) Author to whom correspondence should be addressed. Electronic mail: yep@purdue.edu
}

growth environment is pumped to an approximate pressure of $2 \times 10^{-7}$ mbar before temperature ramping at a rate of $10-20{ }^{\circ} \mathrm{C} / \mathrm{min}$ and up to a specified growth temperature. The growth temperature and time for this particular graphene film are $1550{ }^{\circ} \mathrm{C}$ for $10 \mathrm{~min}$. A room-temperature field-effect mobility measured from graphene grown under these conditions is as high as $5400 \mathrm{~cm}^{2} / \mathrm{V} \mathrm{s}^{8}$

The device structure of the fabricated graphene antidot array is shown in Figs. 1(a) and 1(b). Device isolation and antidot formation of the graphene film are realized by $\mathrm{O}_{2}$ plasma based dry etching with electron-beam lithographically (Vistec VB-6 UHR-EWF) defined hydrogen silsesquioxane (HSQ) resist as the protection layer. The diameter of the holes is around $40 \mathrm{~nm}$ and the defined antidot array period is $80 \mathrm{~nm}$. Ti/Au metallization is used to form the two-terminal Ohmic contacts on a graphene film. Two-point resistance measurements are performed in a variable temperature $(0.4-70 \mathrm{~K}){ }^{3} \mathrm{He}$ cryostat in magnetic fields up to $18 \mathrm{~T}$ using low frequency lock-in technique. The external magnetic field $(B)$ is applied normally to the graphene plane.

Figure 1(c) shows the magnetoconductance $G(B)$ of graphene antidot arrays as a function of perpendicular magnetic field at $0.47 \mathrm{~K}$. The trace is essentially symmetric, $G(B)=G(-B)$, which is the reciprocity relation mandatory for a two-terminal measurement of a stable device. There are three distinguishing features of the measured magnetoconductance. The first is the pronounced weak localization dip around zero magnetic field. The second are distinct conductance minima at \pm 4 and $\pm 8 \mathrm{~T}$. We attribute these minima to the commensurability between the cyclotron orbits of carriers in certain magnetic fields and the period of artificial holes as illustrated in Fig. 1(b). There are three distinct types of carriers involved in magnetotransport in antidot arrays: pinned carries, drifting carries, and scattering carries. ${ }^{1}$ Pinned orbits remain localized about their orbit centers as shown in Fig. 1(b) and cannot contribute to transport. Pinned orbits play a central role here since they remove a fraction of carriers from the transport process. The magnetoconductance shows minima when the carriers with pinned orbits are trapped in this antidot array. Using the commensurability re- 
(a)
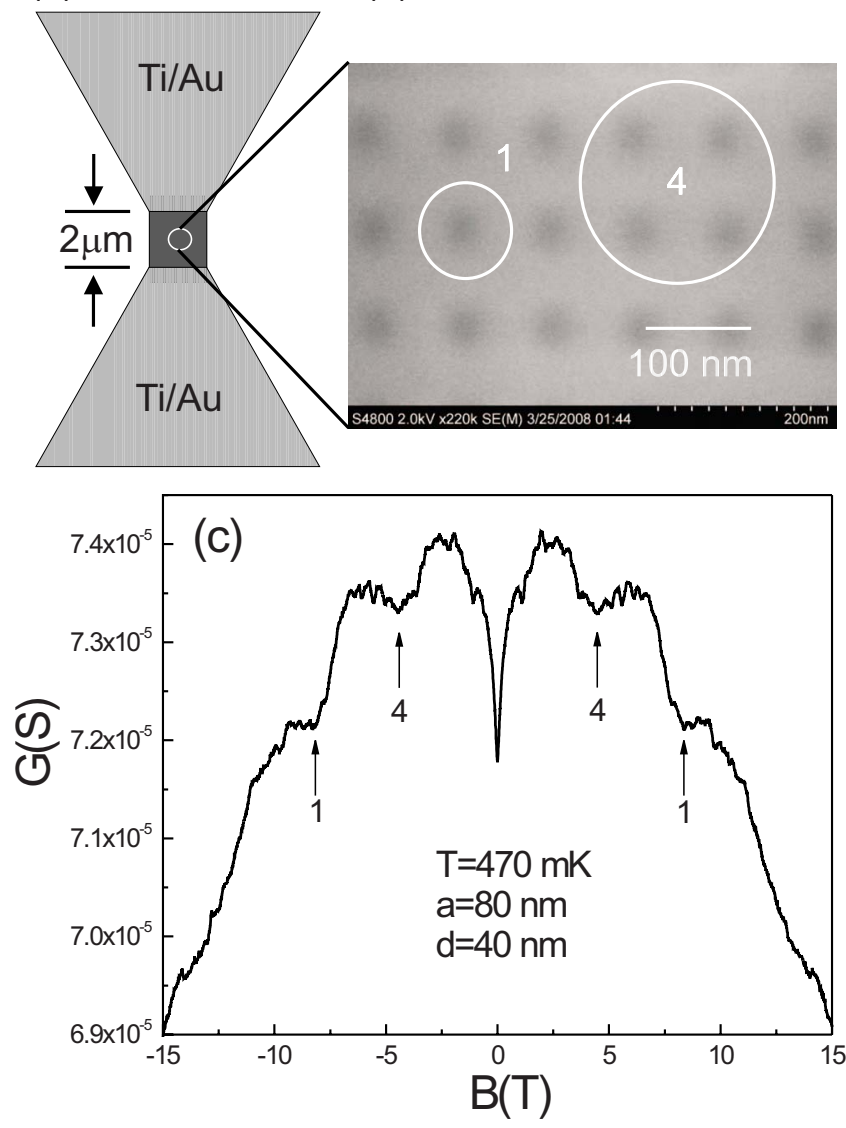

FIG. 1. (Color online) (a) The sample layout with a $2 \times 2 \mu \mathrm{m}^{2}$ graphene area and two-terminal metal contacts. (b) Electron microscopic image of antidote arrays with $\sim 40 \mathrm{~nm}$ holes and $\sim 80 \mathrm{~nm}$ pitches. The commensurate orbits around one antidot and four antidots are sketched to illustrate the physical origin of Weiss oscillations. (c) Magnetoconductance of the graphene antidote arrays measured at $T=477 \mathrm{mK}$. On top of the commensurability oscillations, periodic features are clearly visible as also highlighted in Fig. 2(a).

lation $2 R_{c}=2 \sqrt{\pi N_{s}}(\hbar / e B)=a$, carrier density $N_{s}$ is determined to be $\sim 7.5 \times 10^{12} / \mathrm{cm}^{2}$, where $R_{c}$ is the cyclotron radius, $\hbar$ is the Planck constant, and $a$ is the period of antidot arrays. The elastic mean free path $l_{e}$ is estimated to be $\sim 220 \mathrm{~nm}$ in a reference sample without antidots. The elastic mean free path $l_{e}=2 D / v_{F}$, where carrier diffusive constant $D=E_{F} / 2 N_{s} e^{2} \rho$, Fermi energy $E_{F}=\hbar v_{F} \sqrt{\pi N_{s}}$, and $\rho$ is zerofield resistivity of graphene films. ${ }^{7}$ The $l_{e}$ is larger than the circumference of a single antidot $(\sim 125 \mathrm{~nm})$ but a little bit smaller than the circumference of the central pinned orbits $(\sim 250 \mathrm{~nm})$. It is not a fully ballistic transport from this estimation. The reason for the observation of the second magnetoconductance minima, corresponding to the pinned orbits around four antidots and requiring a much larger $l_{e}$, is not very clear at this moment. The third feature is the tiny structures superimposed on the measured trace, for example, between \pm 2 and $\pm 7 \mathrm{~T}$. Universal conductance fluctuations are suppressed since the sample size $(\sim 2 \mu \mathrm{m})$ is much larger than the phase coherence length. However, quantum interference effect is still observable because the antidot size $(\sim 40 \mathrm{~nm})$ is smaller than the phase coherence length. These tiny periodic features are identified as $\mathrm{AB}$ oscillations, which are related to each magnetic quantum flux penetrating in one antidot cell. AB oscillations on an exfoliated graphene film
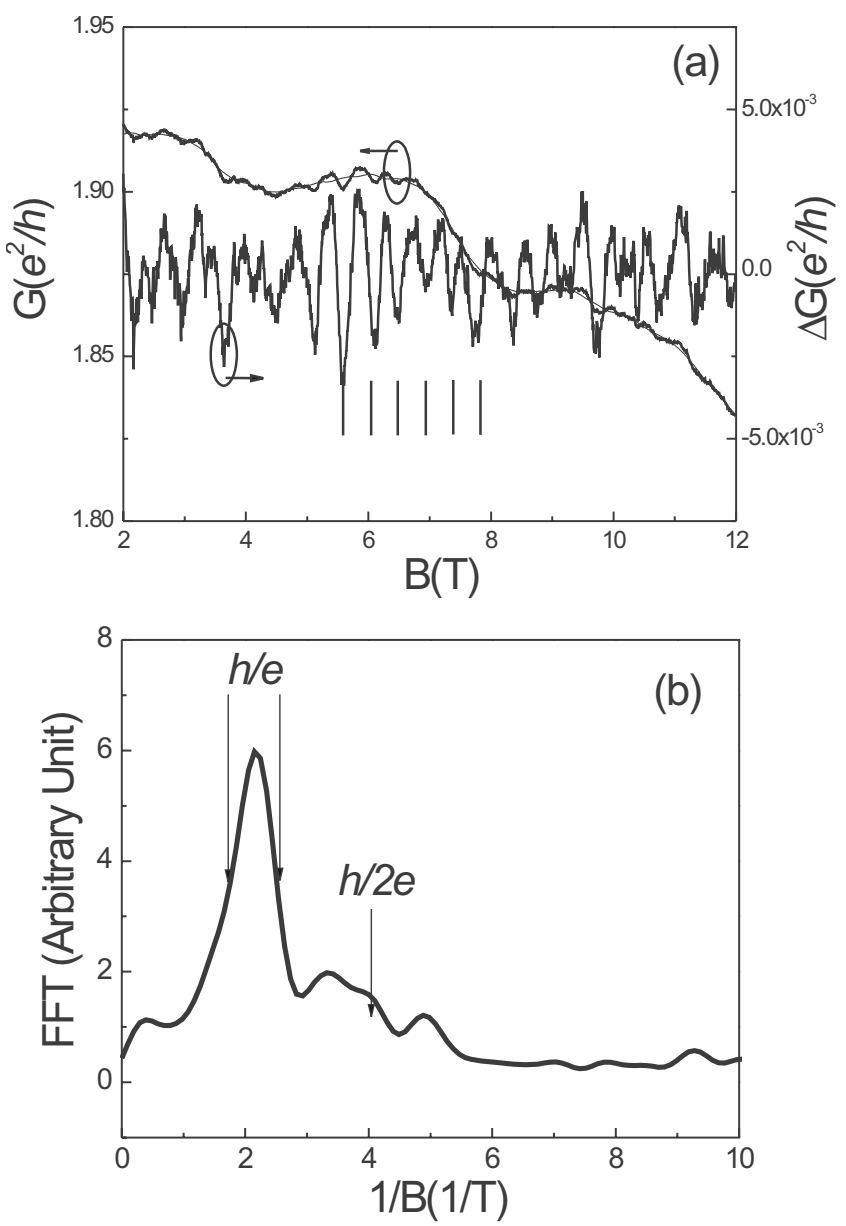

FIG. 2. (a) The solid curve is the measured magnetoconductance. The thin curve is the "baseline" after smoothing the original measured curve. The periodically oscillatory curve is the subtraction of the two black curves. The vertical straight lines are guide to the eyes showing periodic $B$ feature of observed $\mathrm{AB}$ oscillations. (b) Fourier spectrum of the oscillatory gray curve between 2 and $12 \mathrm{~T}$. The solid curve is after six point smoothing. The two vertical straight lines with arrows indicate the positions for half-height of the observed $h / e$ peak, as used for the calculation of the inner and outer radii of the "AB-ring" structure around one antidot.

have been demonstrated experimentally on a single lithographically defined ring. ${ }^{11}$ The conductance fluctuations with the origin of quantum interference effect have also been observed in narrow channeled epitaxial graphene films. ${ }^{7}$

The black thick curve in Fig. 2(a) shows the measured magnetoconductance between +2 and $+12 \mathrm{~T}$ with superimposed oscillatory features. By taking the difference of the measured curve and the black baseline curve obtained from smoothing the measured one, a pronounced $\mathrm{AB}$ periodically oscillatory curve is exhibited as shown as the gray curve in Fig. 2(a). The $\mathrm{AB}$ oscillation period $\Delta B \approx 0.5 \mathrm{~T}$ in this field range is consistent with the condition that the magnetic flux enclosed within the unit cell of the square antidot lattice changes by a single magnetic flux quantum, i.e., $\Delta B$ $=(h / e) / a^{2}$ with $a=80 \mathrm{~nm}$. The rms amplitude of AB oscillations is $\sim 0.01 e^{2} / h$. For detailed discussions, Fig. 2(b) illustrates Fourier power spectrum of Fig. 2(a) with a broad peak centered around $0.47 \mathrm{~T}$, with 0.57 and $0.40 \mathrm{~T}$ as the edges of the half-height width. It corresponds to the inner radius, middle radius, and outer radius of 48,53 , and $57 \mathrm{~nm}$, respectively, if $\Delta B=(h / e) /\left(\pi r^{2}\right)$ where $\pi r^{2}$ is associated with the effective antidot area. It is consistent with the designed ge- 

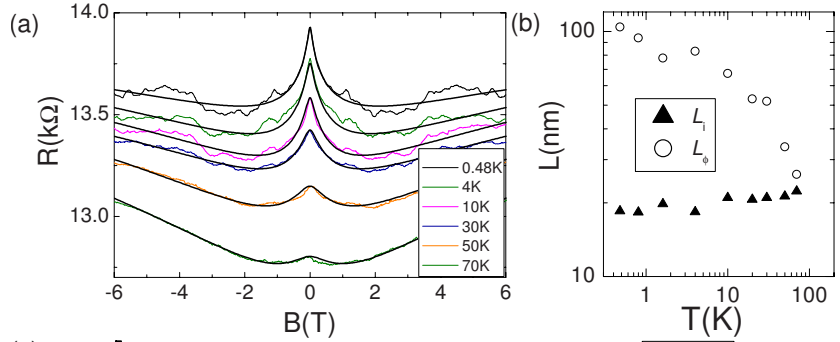

(c)

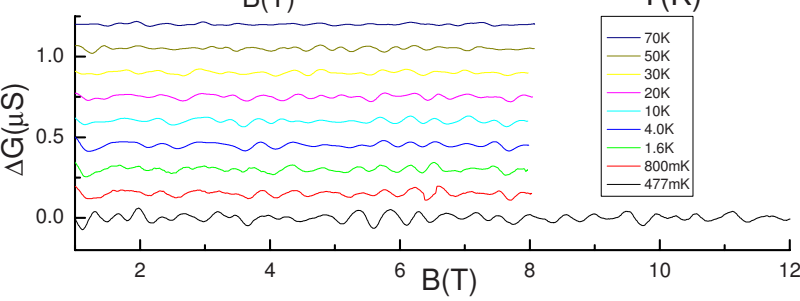

FIG. 3. (Color online) (a) Magnetoresistance as a function of the sample temperature from $477 \mathrm{mK}$ up to $70 \mathrm{~K}$. The solid black curves are fitted weak localization curves in antidot arrays (Ref. 17). (b) The coherence length $L_{\phi}$ and intervalley scattering length $L_{i}$ of the graphene film with antidot arrays vs sample temperatures. (c) Temperature dependence of $\mathrm{AB}$ oscillations. The traces are vertically shifted for clarity.

ometry well with $40 \mathrm{~nm}$ holes and $80 \mathrm{~nm}$ pitches. The relatively large inner radius could be related to overdeveloped resist patterns, plasma overetching, and certain depletion length of graphene edges with unpassivated dangling bonds. The magnetic length $\left(l_{B}=\sqrt{\hbar / e B}=9.2 \mathrm{~nm}\right.$ at $\left.B=6 \mathrm{~T}\right)$ or similar edge channels in the quantum Hall regime could also affect the data. The observed $\mathrm{AB}$ oscillations demonstrate that the epitaxial graphene on $\mathrm{SiC}$ is of high quality and at least has the quantum coherent length larger than $80-100 \mathrm{~nm}$. The weak peak features around $1 / B=4(1 / \mathrm{T})$ could be related to $h / 2 e$ oscillations. ${ }^{3,11}$

While universal conductance fluctuations are generally observed in small graphene flakes, weak localization correction is strongly reduced compared to the conventional $2 \mathrm{D}$ systems due to suppressed backscattering in graphene loosely coupled to the substrate. ${ }^{12-14}$ Short range scattering in epitaxial graphene due to tight binding to the substrate, short range scattering on the edges of antidots, and warping of the Fermi surface at high densities introduces intervalley scattering, ${ }^{15}$ which restores weak localization corrections. ${ }^{16}$ We observe pronounced negative magnetoresistance at low fields with a sharp cusp at zero field characteristic of weak localization in two dimensions, see Fig. 3(a). Moreover, at higher fields, magnetoresistance changes sign, which is expected for the case of strong intervalley scattering. ${ }^{17}$ We used the theory developed in Ref. 17 to analyze the data and extract both phase coherence length $L_{\phi}$ and intervalley scattering length $L_{i}$, see Fig. 3(b). $L_{i}$ is found to be temperature independent and is approximately equal to the distance between antidots, suggesting that scattering on the antidot edges is the dominant intervalley scattering mechanism in our samples. $L_{\phi}$ decreases with the increasing temperature, although it does not follow the $1 / \mathrm{T}$ dependence found in an unpatterned grapheme. ${ }^{15}$ We also note that the range of field where weak localization is observed in an antidot array is much larger than that for the unpatterned samples. The temperature dependence of $\mathrm{AB}$ oscillations is also plotted in Fig. 3(c), which is consistent with the conclusion from Fig. 3(b) by weak localization peak fitting.

In conclusion, we present magnetotransport experiments on antidot arrays fabricated on epitaxially grown graphene films on $\mathrm{SiC}$. The experiment demonstrates the observation of commensurability oscillations and $\mathrm{AB}$ oscillations arising from the artificially imposed lateral potential modulation. The intervalley scattering length and the phase coherence length in graphene antidot arrays are also investigated to explain the temperature dependence of the weak localization and the weak antilocalization.

The authors would like to thank J. A. Cooper, Jr., Y. Lyanda-Geller, M. S. Lundstrom, T. Low, J. Appenzeller, and Kun Xu for valuable discussions, and G. Jones, T. Murphy and E. Palm at the National High Magnetic Field Laboratory (NHMFL) for experimental assistance. Part of the work on graphene was supported by the Nanoelectronics Research Initiative through the Midwest Institute of Nanoelectronics Discovery, The Indiana 21st Century Fund, DARPA, and Intel Cooperation. NHMFL is supported by NSF Grant Nos. DMR-0084173 and ECS-0348289, the State of Florida, and DOE.

${ }^{1}$ D. Weiss, M. L. Roukes, A. Menschig, P. Grambow, K. von Klitzing, and G. Weimann, Phys. Rev. Lett. 66, 2790 (1991).

${ }^{2}$ D. Weiss, K. Richter, A. Menschig, R. Bergmann, H. Schweizer, K. von Klitzing, and G. Weimann, Phys. Rev. Lett. 70, 4118 (1993).

${ }^{3}$ M. Kato, A. Endo, S. Katsumoto, and Y. Iye, Phys. Rev. B 77, 155318 (2008).

${ }^{4}$ A. K. Geim and K. S. Novoselov, Nat. Mater. 6, 183 (2007).

${ }^{5}$ K. S. Novoselov, A. K. Geim, S. V. Morozov, D. Jiang, Y. Zhang, S. V. Dubonos, I. V. Grigorieva, and A. A. Firsov, Science 306, 666 (2004).

${ }^{6}$ Y. Zhang, Y. W. Tan, H. L. Stormer, and P. Kim, Nature (London) 438, 201 (2005).

${ }^{7}$ C. Berger, Z. Song, X. Li, X. Wu, N. Brown, C. Naud, D. Mayou, T. Li, J. Hass, A. N. Marchenkov, E. H. Conrad, P. N. First, and W. A. de Heer, Science 312, 1191 (2006).

${ }^{8}$ Y. Q. Wu, P. D. Ye, M. A. Capano, Y. Xuan, Y. Sui, M. Qi, J. A. Cooper, T. Shen, D. Pandey, G. Prakash, and R. Reifenberger, Appl. Phys. Lett. 92, 092102 (2008).

${ }^{9}$ G. Gu, S. Niu, R. M. Feenstra, R. P. Devaty, W. J. Choyke, W. K. Chan, and M. G. Kane, Appl. Phys. Lett. 90, 253507 (2007).

${ }^{10}$ T. G. Pedersen, C. Flindt, J. Pedersen, N. A. Mortensen, A.-P. Jauho, and K. Pedersen, Phys. Rev. Lett. 100, 136804 (2008).

${ }^{11}$ S. Russo, J. B. Oostinga, D. Wehenkel, H. B. Heersche, S. S. Sobhani, L. M. K. Vandersypen, and A. F. Morpurgo, Phys. Rev. B 77, 085413 (2008).

${ }^{12}$ T. Ando and T. Nakanishi, J. Phys. Soc. Jpn. 67, 1704 (1998).

${ }^{13}$ S. V. Morozov, K. S. Novoselov, M. I. Katsnelson, F. Schedin, L. A. Ponomarenko, D. Jiang, and A. K. Geim, Phys. Rev. Lett. 97, 016801 (2006).

${ }^{14}$ H. Suzuura and T. Ando, Phys. Rev. Lett. 89, 266603 (2002).

${ }^{15}$ A. F. Morpurgo and F. Guinea, Phys. Rev. Lett. 97, 196804 (2006).

${ }^{16}$ F. V. Tikhonenko, D. W. Horsell, R. V. Gorbachev, and A. K. Savchenko, Phys. Rev. Lett. 100, 056802 (2008).

${ }^{17}$ E. McCann, K. Kechedzhi, V. I. Fal'ko, H. Suzuura, T. Ando, and B. L. Altshuler, Phys. Rev. Lett. 97, 146805 (2006). 